\title{
Self-assembled, aligned, and cleaved supramolecules of poly(2,5-pyridinediyl)
}

Matti Knaapila ${ }^{1}$, Mika Torkkeli ${ }^{2}$, Lars-Olof Pålsson ${ }^{3}$, Lockhart E. Horsburgh ${ }^{3}$, Kaija Jokela ${ }^{2}$, Igor P. Dolbnya ${ }^{4}$, Wim Bras ${ }^{4}$, Ritva Serimaa ${ }^{2}$, Gerrit ten Brinke ${ }^{5,1}$, Andrew P. Monkman ${ }^{3}$, and Olli Ikkala ${ }^{1}$

${ }^{1}$ Optics and Molecular Materials, Department of Engineering Physics and Mathematics, Helsinki University of Technology, P.O. Box 2200, FIN-02015 HUT, Espoo, Finland.

${ }^{2}$ Division of X-Ray Physics, Department of Physical Sciences, P.O. Box 64, FIN-00014 University of Helsinki, Finland

${ }^{3}$ Organic Electroactive Materials Research Group, Department of Physics, University of Durham, South Road, Durham, DH1 3LE, United Kingdom.

${ }^{4}$ Netherlands Organisation for Scientific Research (NWO) DUBBLE CRG / ESRF, c/o BP 220 Grenoble F38043, France.

${ }^{5}$ Materials Science Center and Laboratory of Polymer Chemistry, University of Groningen, Nijenborgh 4, 9747 AG Groningen, The Netherlands

\begin{abstract}
We report on directed self-assembly in supramolecules containing rod-like polymers. The comb-shaped supramolecules consist of poly(2,5-pyridinediyl), camphorsulfonic acid, and hydrogen bonded amphiphilic side chains. Locally they form highly ordered lamellar structures in which the polymers are stacked. The liquid crystalline state allows facile overall alignment yielding absorption dichroism and polarized photoluminescence. After alignment, cleavage of the amphiphiles results in solid films which retain this optical anisotropy with the high photoluminescence quantum yield.
\end{abstract}

\section{INTRODUCTION}

Design of self-assembled supramolecules of polypyridines $[1,2]$ is difficult due to their rigid conformation and limited solubility. The structural simplicity and stability make the rod-like poly(2,5-pyridinediyl) (PPY) a good model compound but due to the crystallization [3] and aggregation tendency, both dissolution and alignment of PPY are difficult, as such. PPY can be protonated with sulfonic acids $[4,5]$ of which camphorsulfonic acid (CSA) leads to especially intensive photoluminescence (PL) [4]. In this work, comb-shaped supramolecules of PPY have been developed, based on protonation of pyridine moieties by CSA and hydrogen bonding amphiphilic side groups, resulting in mesomorphism [6]. The side groups may also be regarded as hydrogen bonding solvents and, by varying the composition phases from crystals to disordered liquid can be tailored. In particular, thermotropic liquid crystallinity (LC) is observed allowing facile overall alignment of the chains which leads to high polarisation of absorption and luminescence. In contrast to the covalently bonded side chains [7], the physically bonded side chains can be removed, i.e. cleaved, e.g. by heating under vacuum, based on the dynamic character of the hydrogen bonds. When the amphiphiles are cleaved after alignment in the LC state, the resulting solid material retain the optical anisotropy and the high photoluminescence quantum yield (PLQY) of the pristine polymer [8]. 


\section{EXPERIMENTAL DETAILS}

PPY was first complexed with CSA to form PPY (CSA) $)_{x}$ in $1 \%$ solution of formic acid, followed by rapid evaporation of the solvent and drying. The further side chains were formed by hydrogen bonding amphiphiles 5-pentyl-1,3-dihydroxybenzene i.e. 5-pentylresorcinol (PRES), 4-hexyl-1,3-dihydroxybenzene, i.e.4-hexylresorcinol (HRES), octyl phenol (OP) or octyl gallate, i.e. 1-octyl-3,4,5-trihydroxybenzoate (OG) to this complex. These complexes are denoted as PPY $(C S A)_{x}(\text { amphiphile })_{\mathrm{y}}$ such that $x, y$. are the corresponding molar ratios with the respect to a repeat unit of PPY. Only properly selected $x / y$ ratios are allowed in order to prevent phase separation. The more detailed preparation of the materials can be found elsewhere [6, 8-10].

Structural characterization by X-ray diffraction was carried out using either $\operatorname{CuK}_{\alpha}(\lambda=1.54$ $\AA$ ) radiation from a sealed $X$-ray tube and image plate detector $[8,10]$ or synchrotron radiation at the Dutch-Belgian beamline at ESRF $[6,8,11]$. In order to illustrate the spatial order of the selfassembled lamellae, the normalized correlation function was calculated using equation (1)

$$
\gamma(r)=\frac{\int q^{2} I(q) \cos (q r) d q}{\int q^{2} I(q) d q}
$$

where $I$ is the scattering intensity, $q$ the length of the scattering vector, and $r$ the distance.

Optical absorption spectra were measured using a Perkin Elmer Lambda 19 spectrophotometer $[4,12]$. PL measurements were performed using a charge coupled device (CCD) spectrophotometer (Jobin Yvon Fluoromax) following excitation at the peak of absorption by monochromatized light from a $\mathrm{HgXe}$ lamp. Solid state PLQY measurement [13] were carried out using an integrating sphere mounted in the same device.

\section{RESULTS AND DISCUSSION}

\section{Supramolecules and Self-Assembly}

Packing and molecular order of the supramolecules are achieved by self-assembly as a first step of the process. The supramolecule formation and the uniformity of the material have been suggested by the previous studies based on optical spectroscopy, FTIR, AFM as well as optical microscopy with crossed polarizers and scanning near-field optical microscopy $[6,8,10]$. The self-assembly of the supramolecules has been investigated using X-ray diffraction in bulk $(\sim 1$ $\mathrm{mm})[6,8]$, in cast films $(\sim 10 \mu \mathrm{m})[6]$, and in thin films $(\sim 100 \mathrm{~nm})[8,10]$. Supramolecules form lamellar structures in which the partly protonated polymer chains and amphiphiles alternate and where the rod-like polymer chains are stacked in the lamellae. This structure reveals orthorhombic structure showing $h 00,020,040$, and 004 reflections which correspond to the selfassembled layers, basis vector $\mathbf{a}$, the stacking of main chain stacks, $\mathbf{b}$, and the intrachain repeat unit of the polymer, c, respectively [8].

Supramolecules show several structures, depending on their composition. For example, a disordered (i.e. isotropic) fluid, self-assembled (smectic-like) fluid phase, and self-assembled solid during cooling are observed for compositions $(x / \mathrm{y} \sim 1, \mathrm{x}, y \sim 1)$. Biphasic system is usually seen for higher side group concentrations or if $y / x \geq 2$. In such case, the material is either 
macrophase separated or two coexistent self-assembled phases are seen. In following examples CSA concentration is kept rather small, i.e. $x<1.0$, as this maintains relatively high PL [4].

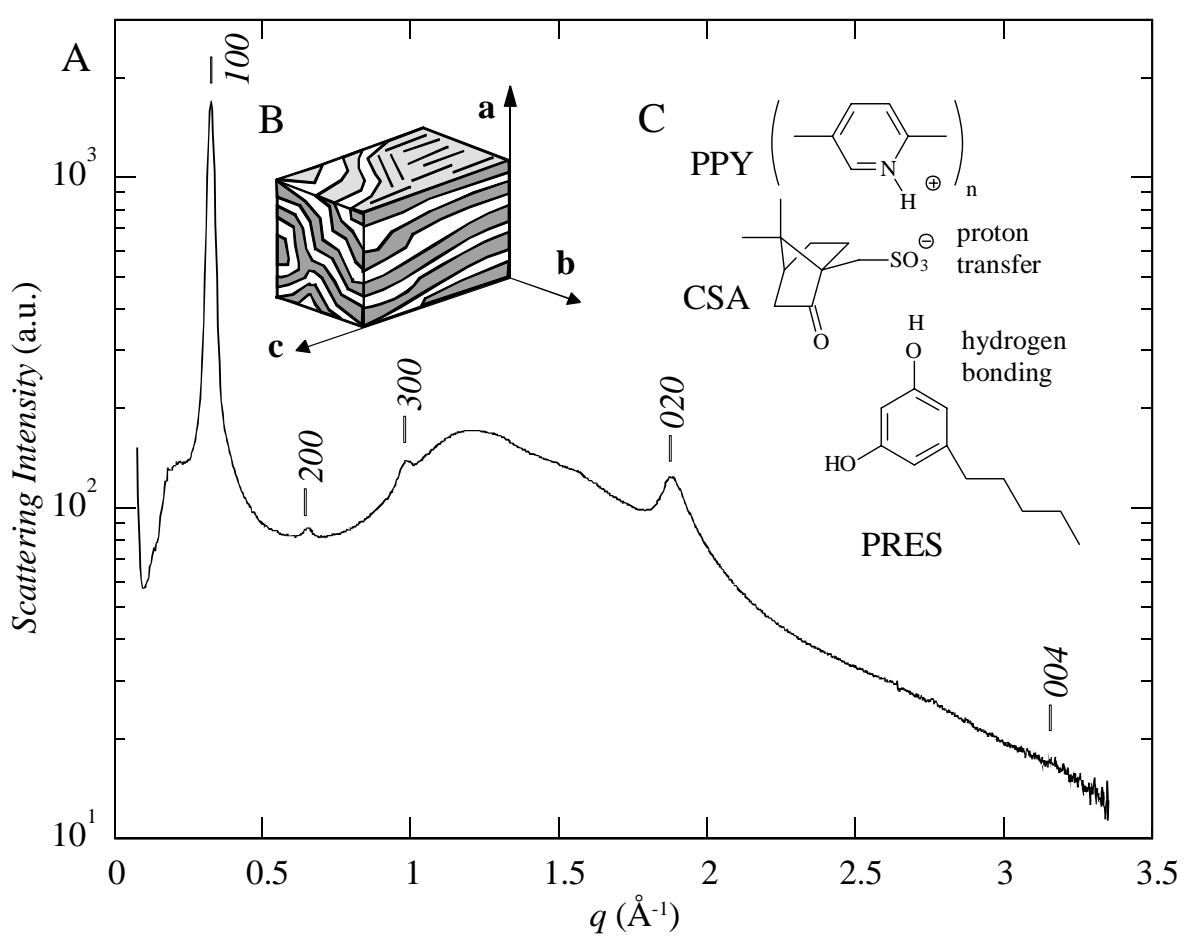

Figure 1. (A) X-ray diffraction pattern of bulk PPY $(\mathrm{CSA})_{0.5}(\mathrm{PRES})_{0.5}$. Inserts: (B) Schematics of lamellar self-assembled structure with alternating polar and nonpolar layers containing PPY within the supramolecules. White layers denote PRES. Current system is defined by orthorhombic lattice with basis vectors $\mathbf{a}, \mathbf{b}$, and $\mathbf{c}$, discussed in text. (C) Chemical formula and possible interactions of the supramolecule. So far, it has not yet been identified where PRES actually forms the hydrogen bonds.

\section{Directed self-assembly i.e. alignment}

The second step involves the control of the overall order of the self-assembled material by shear alignment [8]. The solution cast LC films were sheared in the in-plane direction by drawing them along the smooth substrate surface. The rigid polymers align with their chain axes in the drawing direction and the lamella parallel to the surface. X-ray diffraction patterns were measured ex-situ in the three directions with respect to the aligned sample, see Fig. 2. Because of the alignment, both optical dichroism and polarized PL are observed.

The material shows excellent supramolecular order. The correlation functions normal to lamellae have either a triangular or sinusoidal form (Fig. 2) and damp out very slowly with large coherence length. Depending on the composition, the correlation length deduced from the width of reflection 100 as high as 670-790 ̊ can be achieved [8]. Furthermore, the peak widths of the higher order reflections $h 00$ are nearly linearly dependent on $q$. Therefore, the reflection widths are principally due to lattice parameter fluctuations (microstrains) and the actual crystallite size may be considered far higher. Note that the coherence is related with the spatial order of the local lamellar order. The degree of macroscopic alignment is indicated by the angular spreads of the 
reflections, which also easily gives results that are comparable with the aligned poly(alkylthiophenes) [7], for instance.
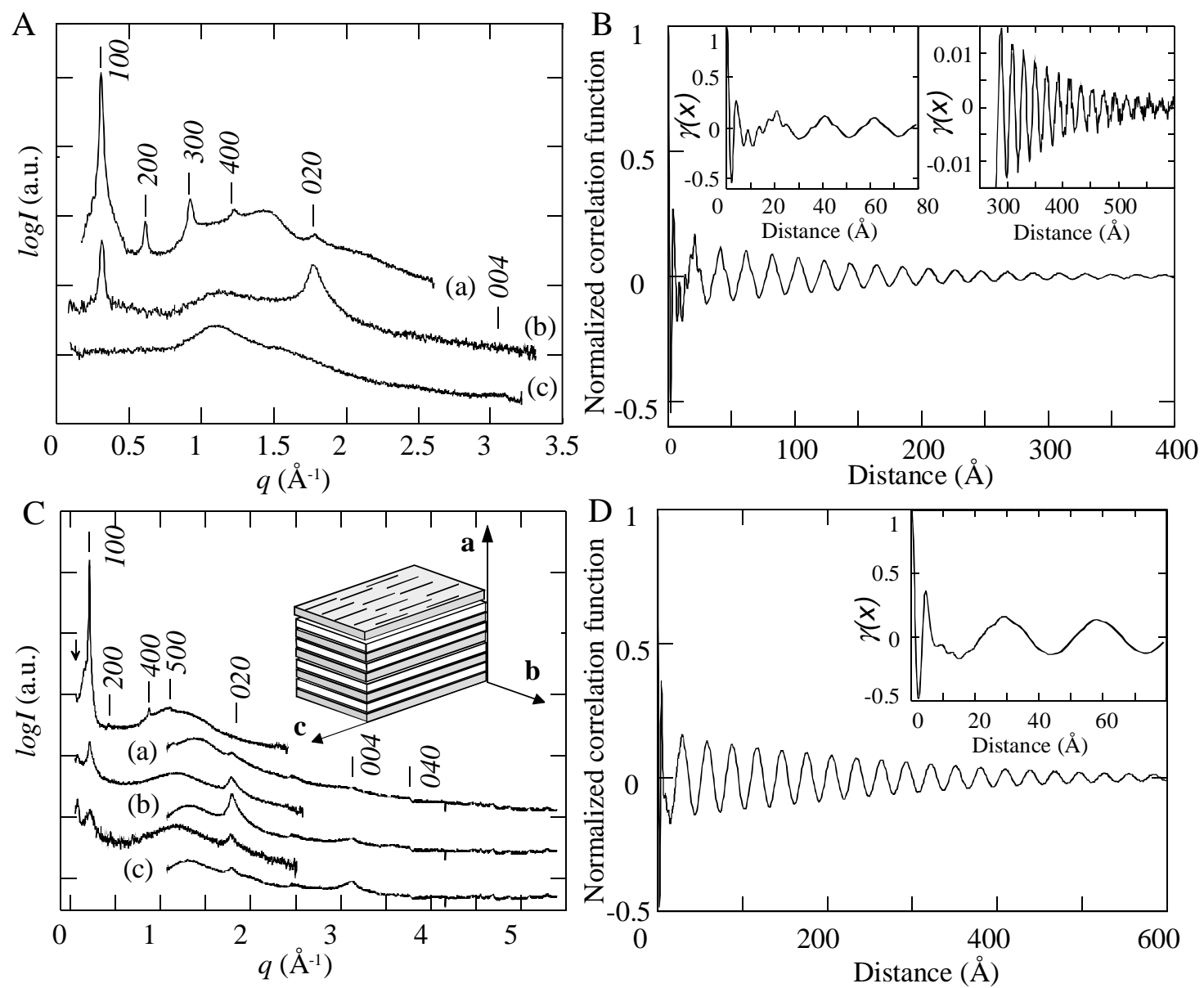

Figure 2. A) Scattering intensity curves for the aligned PPY $(\mathrm{CSA})_{0.5}(\mathrm{PRES})_{0.5}$; B) The corresponding one-dimensional correlation function normal to the lamellae; C) Scattering intensity curves for the aligned PPY $(\mathrm{CSA})_{0.5}(\mathrm{OP})_{1.0}$; D) The corresponding one-dimensional correlation function normal to the lamellae. Curves (a): data normal i.e. equatorial out-of-plane; curves (b): equatorial i.e. equatorial in-plane; curves (c): meridional. Reflection at ca. $0.09 \AA^{-1}$ marked with an arrow is seen along the meridional direction forming horizontal scattering streaks [8], which suggests nematic order, and higher hierarchy level of the structure along the chains. Definition of the directions: equatorial out-of-plane (perpendicular to polymer chain, normal to the surface, a); equatorial in-plane (perpendicular both to polymer axis and surface normal, b); meridional (parallel to the polymer chain axis, $\mathbf{c}$ ). 


\section{Cleavage of the side chains}

As a third step, the hydrogen bonded side chains were cleaved by vacuum treatment $\left(\sim 10^{-3}\right.$ mbar) at elevated temperature. Simultaneously, the PLQY was enhanced and the aligned samples remained optically anisotropic as revealed by dichroism and polarized PL. Therefore, the overall order of PPY is not affected by heating. For example, when a cast film of PPY $(\mathrm{CSA})_{0.5}(\mathrm{HRES})_{0.5}$ was kept at $120{ }^{\circ} \mathrm{C}$ for 42 hours, FTIR spectra reminiscent of PPY(CSA) 0.5 was observed, which suggested the removal of the HRES $\left(T_{m}=68^{\circ} \mathrm{C}\right)$. The PLQY of thin spin-coated film $(\sim 100 \mathrm{~nm})$ increased from $(10 \pm 1) \%$ to $(20 \pm 1) \%$, the latter being at the same level as that of the optically isotropic PPY (CSA $)_{0.5}$ and PPY. In cast films $(\sim 10 \mu \mathrm{m})$, a similar enhancement was seen, although at lower PLQY [8]. Not surprisingly, PRES $\left(T_{m}=48^{\circ} \mathrm{C}\right)$ gave identical results, whereas the less volatile OG $\left(T_{m}=102{ }^{\circ} \mathrm{C}\right)$ could not be removed as efficiently based on FTIR. However, for the cast films of PPY $(\mathrm{CSA})_{0.5}(\mathrm{OG})_{0.5} \mathrm{kept}$ in $120{ }^{\circ} \mathrm{C}$ for 72 hours (Fig. 3), the PLQY increased from ca. $1 \%$ to $(7 \pm 1) \%$. The detailed mechanism of how the PLQY changes remains partially open.

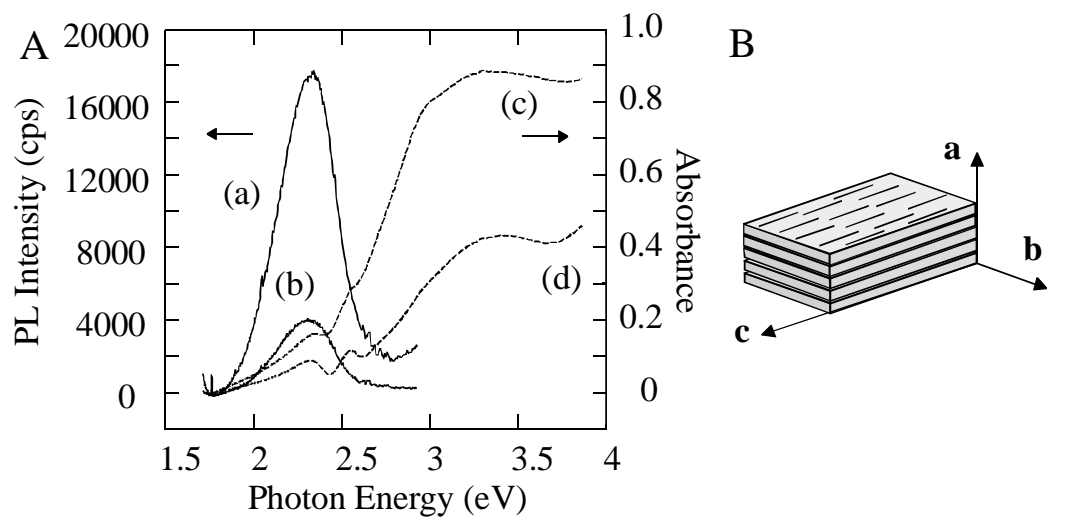

Figure 3. A) Absorption dichroism and polarized PL in aligned films of PPY $(\mathrm{CSA})_{0.5}(\mathrm{OG})_{0.5}$ kept in vac $\left(10^{-3}\right.$ mbar) at $120{ }^{\circ} \mathrm{C}$ for three days, and B) possible schematics. During this process PLQY increased from $\sim 1 \%$ to $(7 \pm 1) \%$. The PL spectra (solid lines) were obtained with the $\mathbf{c}$ axis of the sample in vertical orientation for both curves. Curve (a) was observed when both the excitation light $(\lambda=370 \mathrm{~nm})$ and the detected PL were vertically polarized, parallel to the $\mathbf{c}$ axis. Curve (b) was seen when the excitation and detected PL were both horizontally polarized. The PA curves (dashed lines) were obtained with linearly polarized light. Curve (c) was observed when the $\mathbf{c}$ axis was placed parallel to the polarization vector of the probe light. Curve (d) was seen for their mutual perpendicular orientation. Both results indicate that the transition dipoles are predominantly oriented parallel to the $\mathbf{c}$ axis. Because of similar shape of PL spectra, the origin of PL is suggested to be same both in the case (a) and (b). Slight redshift of PA curve (c) relative to (d) have also been observed in a stretch oriented PPY [12]. The difference in sensitivity of spectrometer was calibrated using dilute Coumarin 314 solution in methanol. 


\section{CONCLUSIONS}

Conjugated rod-like polymer, PPY, forms comb shaped supramolecules which assemble in highly ordered smectic local structures and can be aligned to form directed assembly. High absorption dichroism and polarized PL are observed. Hydrogen bonded amphiphiles can be cleaved after alignment, which retains optical anisotropy and improve PLQY.

\section{REFERENCES}

[1] S. S. Zhu, P. J. Carroll, and T. M. Swager, "Conducting polymetallorotaxanes: A supramolecular approach to transition metal ion sensors," J. Am. Chem. Soc., vol. 118, pp. 8713-8714, 1996.

[2] D. A. P. Delnoye, R. P. Sijbesma, J. A. J. M. Vekemans, and E. W. Meijer, “ $\pi$ Conjugated oligomers and polymers with a self-assembled ladder-like structure," J. Am. Chem. Soc., vol. 118, pp. 8717-8718, 1996.

[3] E. J. Samuelsen, A. P. Monkman, L. A. A. Pettersson, L. E. Horsburgh, K. E. Aasmundtveit, and S. Ferrer, "The structure of polypyridine," Synth. Met., vol. 124, pp. 393-398, 2001.

[4] A. P. Monkman, M. Halim, I. D. W. Samuel, and L. E. Horsburgh, "Protonation effects on the photophysical properties of poly(2,5-pyridine diyl)," J. Chem. Phys., vol. 109, pp. 10372-10378, 1998.

[5] M. Jonforsen, S. Grigalevicius, M. R. Andersson, and T. Hjertberg, "Counter-ion induced solubility of polypyridines," Synth. Met., vol. 102, pp. 1200-1201, 1999.

[6] M. Knaapila, J. Ruokolainen, M. Torkkeli, R. Serimaa, L. Horsburgh, A. P. Monkman, W. Bras, G. ten Brinke, and O. Ikkala, "Self-organized supramolecules of poly(2,5pyridinediyl)," Synth. Met., vol. 121, pp. 1257-1258, 2001.

[7] K. Tashiro, K. Ono, Y. Minagawa, M. Kobayashi, T. Kawai, and K. Yoshino, "Structure and thermochromic solid-state phase transition of poly(3-alkylthiophene)," J. Polym. Sci. B: Polym. Phys., vol. 29, pp. 1223-1233, 1991.

[8] M. Knaapila, O. Ikkala, M. Torkkeli, K. Jokela, R. Serimaa, I. P. Dolbnya, W. Bras, G. ten Brinke, L. E. Horsburgh, L.-O. Pålsson, and A. P. Monkman, "Polarized luminescence from self-assembled, aligned, and cleaved supramolecules of highly ordered rodlike polymers," Appl. Phys. Lett., vol. 81, pp. 1489-1491, 2002.

[9] L. E. Horsburgh, A. P. Monkman, and I. D. W. Samuel, "Synthesis and characterisation of polypyridines," Synth. Met., vol. 101, pp. 113-114, 1999.

[10] M. Knaapila, M. Torkkeli, T. Mäkelä, L. Horsburgh, K. Lindfors, R. Serimaa, M. Kaivola, A. P. Monkman, G. ten Brinke, and O. Ikkala, "Self-organization of nitrogencontaining polymeric supramolecules in thin films," Mat. Res. Soc. Symp. Proc., vol. 660, pp. JJ5.21.1-6, 2001.

[11] W. Bras, "A SAXS/WAXS beamline at the ESRF and future experiments," J. Macromol. Sci. Phys., vol. B37, pp. 557-566, 1998.

[12] F. Feller and A. P. Monkman, "Optical spectroscopy of oriented films of poly (2,5pyridinediyl)," Phys. Rev. B, vol. 61, pp. 13560-13564, 2000.

[13] L.-O. Pålsson and A. P. Monkman, "Measurements of solid-state photoluminescence quantum yields of films using a fluorimeter," Adv. Mater., vol. 14, pp. 757-758, 2002. 